\title{
Design of Coal Mine Power Supply Monitoring System
}

\author{
Lei Shi 1, Guo Jin 2 and Jun Xu 3 \\ ${ }^{1}$ Department of electronic and Information Engineering, Henan polytechnic institute, Henan \\ Nanyang 459000, China \\ ${ }^{2}$ College of mechanical and electrical automation, Henan polytechnic institute, Henan Nanyang \\ 473000, China \\ ${ }^{3}$ Henan Optical Group Co., Ltd., Henan Nanyang 473000, China
}

Keywords: Coal mine, Power supply system, Monitoring system, Fault diagnosis.

\begin{abstract}
According to the characteristics and the management pattern of coal enterprises production and supply, this paper designs a kind of mine power supply monitoring system. The system can carry out real-time monitoring, measurement, protection and control for mine power equipment, the realization of underground high, low-voltage power supply devices can be real-time power data acquisition, data recording, fault diagnosis, fault alarm, fault recording and other large-scale monitoring system of automatic control. The system improves the stability and reliability of mine power system, effectively reducing the occurrence of the mine unplanned power outages, at the same time it also can be very conveniently understanding the on-site power supply system status and failures, which reduce the time to understand the scene, improving the production efficiency.
\end{abstract}

\section{Introduction}

The coal mine power grid monitoring system is based on the characteristics of power supply in our country coal enterprises, it is the application of advanced computer network communication and control technology in the design of management mode, and then the system carries out monitoring, measurement, protection and control for the mine power equipment [1-3]. For the mine high and low voltage power supply equipment, the system can carry on real-time electric power data acquisition, data recording, fault diagnosis, fault warning, fault record and other automatic control's large-scale monitoring system $[4,5]$. Using the system can achieve the ground control center on the underground high voltage power supply equipment's four remote functions of telemetering, remote adjustment, remote debugging and remote control, so that the stability and reliability of mine power system is greatly improved, to effectively reduce the occurrence of mine unplanned blackouts. At the same time, the system can generate a variety of records and statistical reports [6-8]. For the information of display and record power supply system, people are very convenient to know the situation of the power supply system and the fault, in order to timely inform the relevant departments to handle whether people are in control or in the office, people can carry out interactive operation, lets operators and managers to make efficient decisions, to realize the monitoring and management of underground power supply system, to reduce the field solution time, and the method improves the production efficiency.

\section{System Components}

The coal mine power supply monitoring system is a variety of mine mechanical and electrical control equipment and PC monitoring software components, such as ground center station, mine flameproof and intrinsically safe data interface, mine flameproof and intrinsically safe power monitoring and control substation, mine flameproof and intrinsically safe type DC regulated power supply, remote control transmitter, high and low voltage vacuum electromagnetic device, distribution device and so on, and it is shown in Figure 1.

Mine electric power safety monitoring master station. Master station system is the control and management center of distribution automation system in coal mine, which is mainly composed of channel cabinet, data interface (communication management), data server, monitoring workstation 
and network equipment. The system uses the structure of distributed system, it provides various forms of organization, it can be stand-alone systems, and it is also a multi machine system [9-10]. The system carries out the remote online real-time monitoring for all substations, to achieve record events, all kinds of curve statements' storage retrieval and print, protection fixed value remote setting and remote operation control, electricity metering management, fault record wave storage analysis and printing etc.. The system's high precision measuring and monitoring, professional chart analysis tools as well as recording and analysis tools is the new modern tools of strengthening power supply management, reducing accidents, reducing loss, saving electric energy, improving the operation efficiency and management level, to provide a guarantee for the realization of unattended substation or fewer people on duty. For the KJ172 power monitoring master station system as a regional control center, we can be further as a superior integrated system, and it can further be used as a node and superior system networking of the superior integrated system, to carry out information exchange and sharing with the superior system.

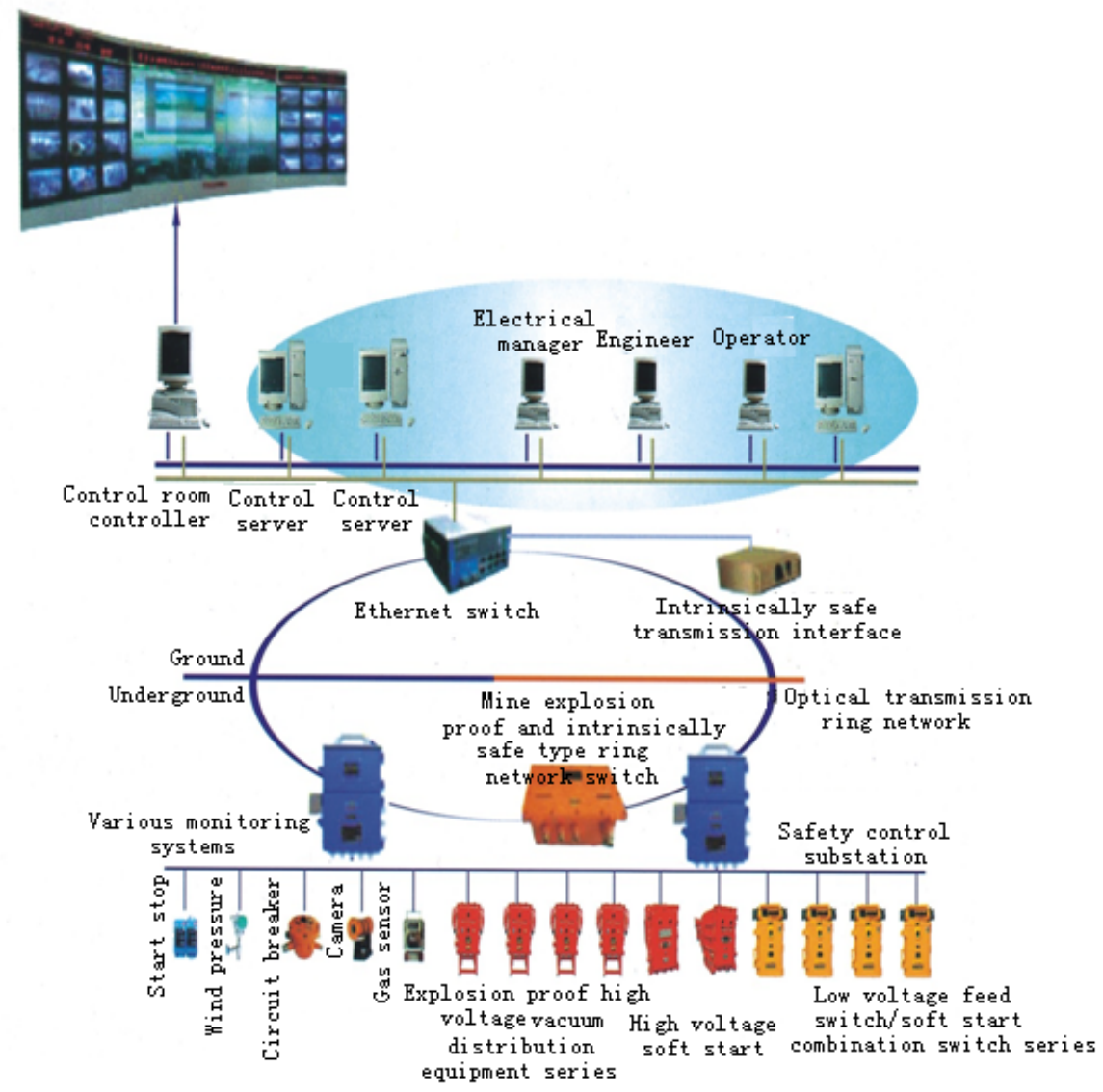

Fig. 1 The composition of coal mine power supply monitoring system

Mine flameproof and intrinsically safe power substation. Substation is the middle part of the coal mine electric power safety monitoring system. Between the power monitoring master station and distribution equipment, it not only can realize distribution equipment's telemetry, remote communication, remote control, electric measurement, protection parameter adjustment and other data collection and forwarding function within the jurisdiction, but also can prevent the leapfrog trip network monitoring and analysis, leakage grounding line selection etc..

The system uses the Intel Core 2 Duo E5700 3.0GHZCPU, 2G DRAM memory and 32M FLASH; it uses LINUX embedded real-time operating system and C programming language; its hardware and software are modular configuration, to facilitate the expansion and upgrading of the system; it is the built-in 1.5KV electromagnetic isolation protection; there are 6 standard serial port, RS232/485/422 software, 2 CAN2.0 interface, 2 10/100M Ethernet port /SC port optional, ESD protection and protection signal $15 \mathrm{kV}$; its working temperature is $-40-75^{\circ} \mathrm{C}$; it can access a variety of communication protocols and provides a flexible protocol conversion program; its LCD super 
widescreen color design is easy to manipulation and real time view of all the switch operation information, which is suitable for unattended substation.

Mine flameproof and intrinsically safe data interface. Data interface (fiber optic network switches) can be composed of dual ring self-healing optical fiber network, it mainly completes the data transmission between ground and underground, substation and substation, and it can access to the Internet video and other network equipment, which main bears data transmission business between ground and underground, different equipment.

Data interface provides 2 pairs of redundant 100Base-FX full duplex single mode fiber interface, and using these optical fiber interfaces can be composed of optical fiber redundant ring. When a point equipment or optical cable in the network has a failure, the network will resume communication within the 300ms. At the same time, the data interface can realize data exchange between 2 Ethernet optical signal and 6 Ethernet electrical signals, it has bidirectional communication and indication function with the substation equipment and the host device, and it has fault diagnosis and indication function. At the same time, it has power source indication and external standby power supply, external KDW660/24B mine flameproof and intrinsically safe type DC regulated power supply provides the backup power continuous time (full load) that will be not less than 2 hours after the power outage.

Distribution terminal equipment layer. This system arranges the intelligent microcomputer monitoring protector switch in the high and low-voltage explosion-proof feed switch, it not only can complete the high and low pressure feed line's voltage, current, active power, no power, power factor, power supply, switch on/switch state power parameter detection, but also can complete the underground intelligent explosion proof switch's switch/closing control, parameter setting and fault diagnosis and other functions.

A new generation of intelligent switch microcomputer monitoring and protection device uses 32 DSP high speed digital signal processor, special electric metering unit, differential network interface and industrial grade high accuracy 16 synchronous sampling A/D. Based on HYZB-GT type intelligent switch microcomputer monitoring and protection device, it also increase the short-circuit protection anti override trip, multi rate multi-functional power measurement, equipment monitoring and environmental temperature monitoring, it further realizes the monitoring data real-time dynamic show, time management of electricity metering, line and equipment failure's warning, rapid positioning and accurate resection, sequential events' accurate record and other functions, which lays the foundation for the realization of the power supply system automation operation and information management.

Mine flameproof and intrinsically safe DC power supply. Mine flameproof and intrinsically safe DC power supply can not only provide 24V DC power supply and backup power supply for underground monitoring sub stations, data interface and other devices, but also can provide $18 \mathrm{~V}$ or $12 \mathrm{~V}$ output intrinsic safety power supply for coal mine intrinsic safety type low load equipment.

\section{System Scheme Design}

According to the actual situation of power monitoring system, the master can be divided into dual network server mode configuration and single network configuration mode. Dual network dual server configuration mode is suitable for construction in electric power safety monitoring system as the core of the integrity of the information management center, which is divided into electric power monitoring station, monitoring substation and power distribution terminal three layer structure. The model can best reflect the superiority of the model for the network structure of mine multi ring network power supply, as shown in Figure 2. 


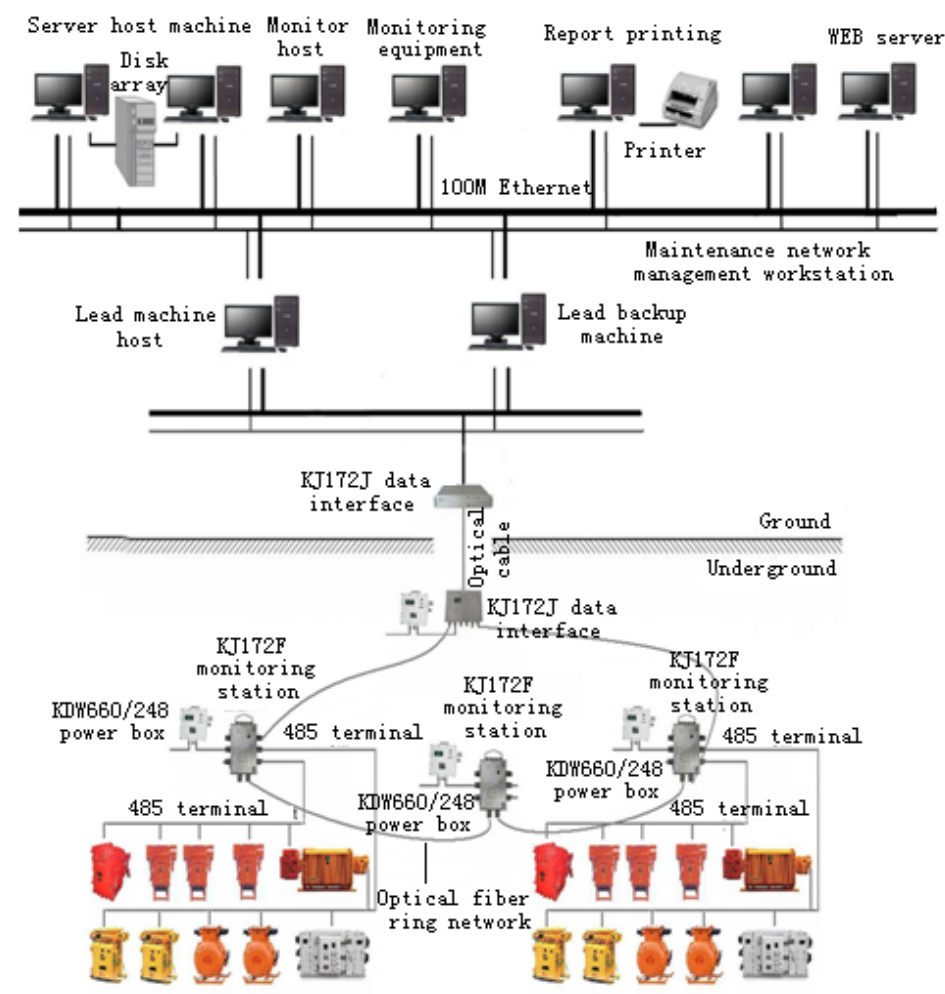

Fig.2 Dual network dual server configuration

Single network configuration mode is suitable for the shortage of funds or system scale coal mining enterprises, the system configures the main standby monitoring station, remote viewing workstations and other necessary equipment, and it is shown in Figure 3. In the minimization scheme configuration of the power master station monitoring system, it can be configured even only 1 computer to complete the necessary functions of the entire system.

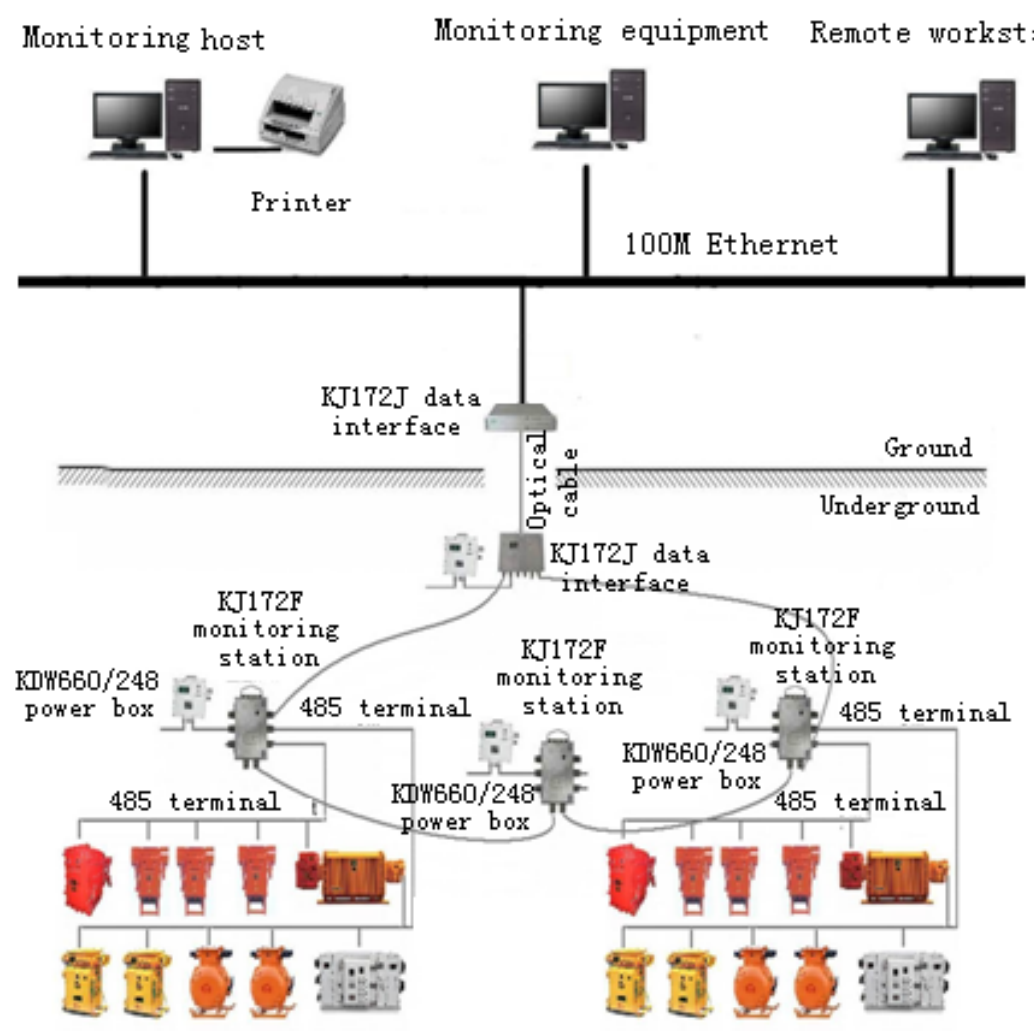

Fig.3 Single network dual server configuration 
Coal mine electric power monitoring main station system function. Coal power monitoring master station system includes power SCADA, statistical calculation and analysis, Web and other functions. Power SCADA function mainly includes data acquisition and display (curve, report and chart), switching control operation, setting value adjustment, data exchange and transmission, man-machine interface (system simulation diagram, power network topology and system operation picture), graphics editing (system simulation diagram, network topology and system operation picture), incident/accident alarm, authority management, historical data management (curve, report, fault alarm recording and playback), print output, system management monitoring, equipment (equipment information query and maintenance records), channel monitoring and station system maintenance; The statistical calculation and analysis function mainly includes switch frequency statistics, the qualified rate of the voltage statistics, power supply reliability statistics, prevent tripping fault analysis, electric energy metering and load analysis and other functions; Web remote browsing function mainly includes WebSCADA and Web statements. Through the WEBSCADA, we can not only browse the distribution network logic structure diagram, substation main wiring simulation diagram, history curve and voltage bar chart in the remote browsing workstation, but also can browse real-time data. Through the WEB report, the remote users can browse the system maintenance log and the power supply distribution equipment accounting data. For users, remote workstation can only browse through the WEB function data, and it cannot be controlled by the WEB operation, to ensure the safety of the system.

Coal mine electric power monitoring master station system software. The interface of database management software is a "Windows" window, the title bar is the name of the software "database management system", the menu line lists all the function and options of the software, the state line indicates whether allow you to modify state parameters, and the toolbar is equivalent to the commonly used menu shortcut keys, it can be quickly transferred out of commonly used functions. As a matter of fact, all the tool bar function is included in the menu. After entering the Windiws98/NT/2000/XP computer, the system firstly runs KDZK.exe, and then runs KDDB.exe, finally to enter the main interface of the system.

\section{Summary}

This paper uses the advanced computer network communication and control technology to carry out monitoring, measurement, control and protection for mine power equipment, realizing the real-time power data acquisition, data recording, fault diagnosis, fault warning, fault recording and automatic control's large-scale monitoring system on high and low voltage power supply devices, this system has good real-time performance, and its monitoring and control information feedback are timely and accurately. The system software adopts advanced program language, it has graphical user interface, good operating platform and powerful function, it is easy to use, it also supports Web browsing, and because of stable and reliable performance, the user can be the design of the distribution with the power supply network change. At the same time, the system has good scalability, and it uses the tree structure, so it has strong adaptability, which can meet the needs of coal mine multi branch power supply network. The system has passed the tests of national safety production equipment inspection center in Shanghai mine and the national safety inspection center, and it has been put into use in mine.

\section{References}

[1] X.G. Guo. Research status and development trend of mine power supply monitoring system. Coal mining machinery, 2014, 35(10): 118-120.

[2] R. Fan. Coal mine power supply monitoring system and its development trend. Industry and mine automation, 2014, 36(8): 30-34.

[3] X.R. Liu, Y.L. Zhao, Z.T. Feng. Application of mine power monitoring system based on fieldbus. Shandong coal science and technology, 2014(5): 57-58. 
[4] G. Wang. Design and application of mine power supply remote monitoring and control system. Mechanical and electrical technology, 2014(5): 76-79.

[5] H.F. Chen. Design of mine main fan monitoring system. Industrial automation, 2014(9): 103-105.

[6] X.K. Li, Q.B. Su. Application of KJ316 Coal Mine Power Supply Monitoring System. Value engineering, 2013(22): 233-234.

[7] H. Wang. Mine power monitoring and dispatching optimize system based on Web. Coal mine machinery, 2015, 36(7): 290-291.

[8] C.Y. Zhang, F.Z. Wang. The mine underground power supply remote monitoring system based on RS-485 bus. Mining machinery, 2013(5): 120-121.

[9] S.Y. Yu. Design of mine power supply monitoring system. Small and medium- sized enterprise management and technology, 2013(10): 206-207.

[10] Q.Y. Zhu, B.W. Gao. Design and application of coal mine power network monitoring and control system. China coal, 2014, 35(9): 68-70. 